\title{
DUXAP8 knockdown inhibits the development of melanoma by regulating the miR-3182/NUPR1 pathway
}

\author{
XIGE CHEN $^{1}$, JUAN GAO ${ }^{1}$ and NING LIANG ${ }^{2}$ \\ ${ }^{1}$ Department of Dermatology, Weihai Central Hospital, Weihai, Shandong 264400; ${ }^{2}$ Department of Dermatology, \\ The Affiliated Changzhou No. 2 People's Hospital with Nanjing Medical University, Changzhou, Jiangsu 213000, P.R. China
}

Received July 28, 2020; Accepted March 22, 2021

DOI: $10.3892 / \mathrm{ol} .2021 .12756$

\begin{abstract}
Double homeobox A pseudogene 8 (DUXAP8) has been reported to regulate the growth of several types of cancers, such as breast cancer and ovarian cancer. However, its role in melanoma remains unclear. In the present study, the mechanism through which DUXAP8 regulates melanoma progression was explored. The expression levels of DUXAP8 were determined in 43 samples from patients with melanoma in different stages, as well as human epidermal melanocytes cells and malignant melanoma cell lines using reverse transcription-quantitative PCR (RT-qPCR). The prognosis of patients was analyzed using the Kaplan-Meier method. The relationship between lncRNA DUXAP8 expression and microRNA (miR)-3182 or nuclear protein 1 transcriptional regulator (NUPR1) levels was analyzed using Pearson's correlation. Luciferase reporter and RNA pull-down were used to examine the interactions between these molecules. Proliferation was assessed using Cell Counting-Kit-8. Transwell assays were used to examine cell migration and invasion. lncRNA DUXAP8 was upregulated in melanoma tissue and cells compared with normal tissues and cells. The levels of DUXAP8 inversely correlated with survival time of patients with melanoma. Knockdown of lncRNA DUXAP8 inhibited proliferation, migration and invasion of melanoma cells. lncRNA DUXAP8 targeted miR-3182, while miR-3182 targeted NUPR1. The overexpression of NUPR1 reversed the effects of DUXAP8 knockdown or miR-3182 mimic on melanoma progression. In conclusion, lncRNA DUXAP8 downregulation inhibits the development of melanoma by regulating the miR-3182/NUPR1 axis.
\end{abstract}

Correspondence to: Dr Ning Liang, Department of Dermatology, The Affiliated Changzhou No. 2 People's Hospital with Nanjing Medical University, 29 Xinglong Lane, Changzhou Jiangsu 213000 , P.R. China

E-mail: ning_liangzzz@163.com

Key words: melanoma, DUXAP8, microRRNA-3182, NUPR1

\section{Introduction}

Melanoma is one of the most common malignant tumors and its incidence increases by 3-5\% percent annually worldwide (1). The common pathological types of melanoma are superficial spreading type, nodular type, malignant freckles and acral freckles (2). Superficial spreading type is the most common in the white ethnicity, and extremity freckle-like melanoma is more common in Asians and Pacific Islanders (3). The 5-year survival rates of patients with melanoma at stage I, II, III and IV in China are 94, 44, 38 and 4.6\%, respectively (4). Moreover, the survival rate is also closely related to primary ulcers (5).

Excessive ultraviolet radiation is one of the known causes of skin melanoma, and the effects of other factors such as endocrine, chemical and physical factors on the occurrence of melanoma are poorly understood (6). At present, several treatment strategies for melanoma exist (7). Chemotherapy alone has limited efficacy and is hindered by drug resistance (8). Molecular targeting drugs and immunotherapy drugs have made great progress in the treatment of tumors due to their specificity, effectiveness, good patient tolerance and low adverse reactions compared to cytotoxic drugs (9). Multivariate analysis of the relationship between genetic variation and survival revealed that KIT and BRAF gene mutations are independent prognostic factors for melanoma, with risk factors of 1.989 (95\% CI, 1.263-3.131) and 1.536 (95\% CI, 1.110-2.214), respectively (10). However, distant metastases can occur early in malignant melanoma, which is a key factor affecting clinical treatment and leading to poor prognosis (11).

The mechanism of melanoma progression has not been fully elucidated. In addition, research on the mechanism underlying chemotherapy resistance is also of particular interest. It is also worth mentioning that a large part of current research on melanoma is carried out at the genomic level, and less attention has been given to gene expression analysis (12). Studying long non-coding RNA (lncRNA) molecules related to the pathogenesis and progression of melanoma may provide insight into the mechanism of melanoma onset, which is of great significance for the treatment and new drug development. So far, several lncRNA candidates have been explored. Schmidt et al (13) demonstrated that the lncRNA SRA-like non-coding RNA1 could increase the expression of matrix metalloproteinase-9, accelerate invasion of melanoma and 
predict poor survival. Furthermore, homeobox D-antisense1 was confirmed to be upregulated in patients with melanoma and was also closely associated with survival time (14). Although DUXAP8 has been shown to regulate hepatocellular carcinoma and lung cancer development $(15,16)$, its role in melanoma has not been reported. Hence, the present study aimed to investigate whether DUXAP8 is involved in melanoma development and the mechanism of 1ncRNA DUXAP8 on the invasion and metastasis of melanoma was studied, with the aim of identifying a potential molecular-level treatment against melanoma.

\section{Materials and methods}

Patients and tissue samples. A total of 43 patients with skin melanoma (mean age, 52 years; age range, 39-61 years; 27 male patients and 16 female patients) were enrolled from January 2014 to December 2018 at The Affiliated Changzhou No. 2 People's Hospital with Nanjing Medical University (Changzhou, China) or Weihai Central Hospital (Weihai, China). All patients were diagnosed pathologically by at least 3 experienced and independent pathologists from Weihai Central Hospital or the Affiliated Changzhou No. 2 People's Hospital with Nanjing Medical University. Patients who had radiotherapy or chemotherapy before surgery were excluded. Patients that had not received radiotherapy or chemotherapy prior to sample collection were included. The cases included 19 patients at stage I+II and 24 patients at stage III+IV. Tissue samples excised during the operation were immediately frozen in liquid nitrogen until RNA extraction. Adjacent normal tissue was used as a control. The distance between the resected tumor tissue and the healthy tissues was $2 \mathrm{~cm}$. This study was approved by the ethics committee of The Affiliated Changzhou No. 2 People's Hospital with Nanjing Medical University. The patients signed informed consent forms.

Cell lines and transfection. The human epidermal melanocyte HEMa-LP cell lines and the malignant melanoma A375, A2058 and SK-MEL-2 cell lines were purchased from the Cell Bank of Type Culture Collection of the Chinese Academy of Sciences. Cells were cultured in DMEM medium containing 10\% fetal bovine serum (Gibco; Thermo Fisher Scientific Inc.) and $1 \%$ penicillin-streptomycin. The cells were incubated at $37^{\circ} \mathrm{C}$ in an incubator with $5 \% \mathrm{CO}_{2}$.

The eukaryotic expression plasmid vector pSilencerTM3.1-H1 neo containing DUXAP8-shRNA (5'-GGA ACTTCCCAAACCTCCATGATTT-3') and control shRNA (5'-TTCTCCGAACGTGTCACGTTT-3') were constructed by Shanghai GenePharma Co., Ltd. The miR-3182 mimic (5'-GCUUCUGUAGUGUAGUC3'-), NC mimic (5'-UUCUCC GAACGUGUCACGUTT-3'), miR-3182 inhibitor (5'-GAC UACACUACAGAAGC-3'), NC inhibitor (5'-CAGUACUUU UGUGUAGUACAA-3') and NUPR1 overexpression vector (oeNUPR1; pcDNA3-NUPR1) were designed and synthesized by Shanghai GenePharma Co., Ltd. Cells were seeded in 6-well plates. pcDNA3 (Invitrogen; Thermo Fisher Scientific Inc.) empty vector was used as the negative control for oeNUPR1. Transfection was performed using Lipofectamine ${ }^{\circledR} 2000$ reagent (Invitrogen, Thermo Fisher Scientific, Inc.) at $37^{\circ} \mathrm{C}$ for $48 \mathrm{~h}$ with a $100 \mathrm{nM}$ of the aforementioned plasmids, negative controls, mimics and inhibitors, according to the manufacturer's protocol. Transfection efficiency was measured by RT-qPCR and after $48 \mathrm{~h}$ of transfection, subsequent experimentation was performed.

Reverse transcription-quantitative PCR (RT-qPCR). Total RNA was extracted from tissues and cells using TRIzol $^{\circledR}$ (Invitrogen; Thermo Fisher Scientific, Inc.). Total RNA (500 ng) was reverse transcribed into cDNA using SuperScript III (Invitrogen; Thermo Fisher Scientific, Inc.) at $37^{\circ} \mathrm{C}$ for $15 \mathrm{~min}$. Subsequently, qPCR was performed using an ABI PRISM 7500 Sequence Detection system (Thermo Fisher Scientific, Inc.) and SYBR Select Master mix (Thermo Fisher Scientific, Inc.). U6 or GAPDH were used as the internal reference genes. The following thermocycling conditions were used for qPCR: i) Initial denaturation at $95^{\circ} \mathrm{C}$ for $5 \mathrm{~min}$; ii) 40 cycles at $95^{\circ} \mathrm{C}$ for $20 \mathrm{sec}, 58^{\circ} \mathrm{C}$ for $30 \mathrm{sec}$ and $74^{\circ} \mathrm{C}$ for $30 \mathrm{sec}$; and iii) final extension step at $72^{\circ} \mathrm{C}$ for $5 \mathrm{~min}$. The primer sequences were as follows: i) DUXAP8 forward, 5'-ACCCAAACACTAATTGTAGACT-3' and reverse, 5'-TGT CTGGGAGACTGCTTACA-3'; miR-3182 forward, 5'-CAC TCAGCTGGCTTCTGTAGTG-3' and reverse, 5'-CTGGTG TCGTGGAGTCG-3'; NUPR1 forward, 5'-AGGACTTATTCC CGCTGACTGA-3' and reverse, 5'-TGCCGTGCGTGTCTA TTTATTG-3'; GAPDH forward, 5'-GTCGATGGCTAGTCG TAGCATCGAT-3' and reverse, 5'-TGCTAGCTGGCATGC CCGATCGATC-3'; and U6 forward, 5'-CTCGCTTCGGCA GCACATATACT-3' and reverse, 5'-ACGCTTCACGAATTT GCGTGTC-3'. miRNA and mRNA expression levels were quantified using the $2^{-\Delta \Delta \mathrm{Cq}}$ method (17).

Cell-Counting Kit-8 (CCK-8) assay. After cell transfection for 48 h, A375 and A2058 cells were seeded in a 96-well cell culture plate at a density of $2 \times 10^{3}$ cells/well. After incubation for 24,48 or $72 \mathrm{~h}, 10 \mu \mathrm{l}$ of CCK-8 reagent (Beyotime Institute of Biotechnology) was added to each well and incubated for $4 \mathrm{~h}$. The absorbance at $450 \mathrm{~nm}$ was measured using a microplate reader (Bio-Rad Laboratories, Inc.). The average value of each well was taken to evaluate the cell proliferation.

Transwell assay. Cell migration and invasion were assessed by a Transwell assay. For the migration assay, $2 \times 10^{4}$ A 375 or A2058 cells were diluted in FBS-free medium and seeded into the upper chamber (BD Biosciences). For the invasion assay, cells were added onto a Matrigel ${ }^{\circledR}$-pre-coated (Sigma-Aldrich; Merck KGaA) upper chamber. Subsequently, $500 \mu 1$ of culture medium containing $10 \%$ FBS was inoculated into the lower chamber. After $24 \mathrm{~h}$, non-migratory/-invasive cells were detached while the migrated/invaded cells in the lower chamber were fixed in $4 \%$ paraformaldehyde for $30 \mathrm{~min}$ at room temperature and stained using $0.5 \%$ crystal violet for $30 \mathrm{~min}$ at room temperature. The numbers of migratory/invasive cells were counted in five randomly selected fields using an inverted light microscope (Olympus Corporation; magnification, $\mathrm{x} 200)$.

RNA pull-down. Biotin-labeled wild-type (WT) or mutant miR-3182 (Shanghai GenePharma Co., Ltd.) were synthesized. A375 and A2058 cells were lysed using specific lysis buffer (Sigma-Aldrich; Merck KGaA) containing RNase inhibitor 
A

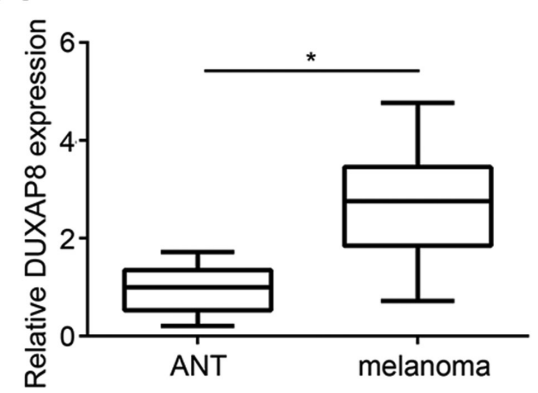

C

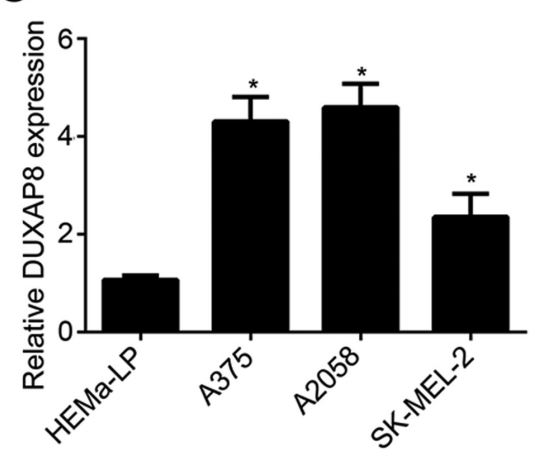

B

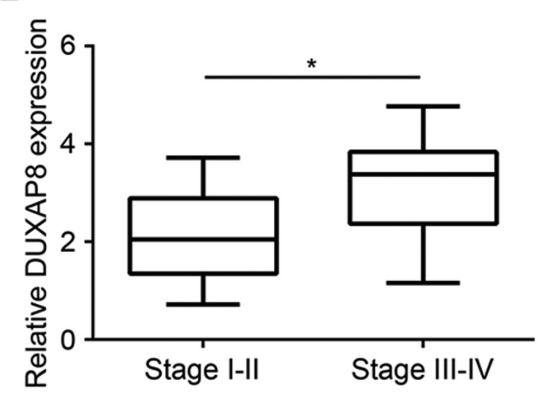

D

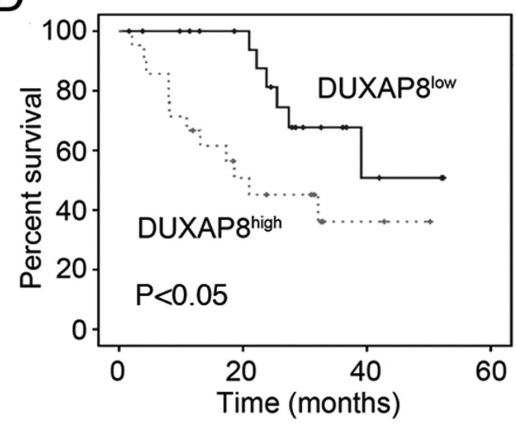

Figure 1. Expression of DUXAP8 and survival analysis. (A) Analysis of DUXAP8 expression levels in 43 pairs of melanoma samples. (B) DUXAP8 expression in samples from patients with melanoma at different stages. I+II stage, $n=19$; III+IV stage, $n=24$. (C) DUXAP8 expression in melanoma cell lines. (D) Survival curve analysis. DUXAP8 ${ }^{\text {high }}, \mathrm{n}=22$; DUXAP8 ${ }^{\text {low }}, \mathrm{n}=21$. ${ }^{*} \mathrm{P}<0.05$. ANT, adjacent normal tissue; DUXAP8, double homeobox A pseudogene 8 .

(Beyotime Institute of Biotechnology). The lysate was subjected to centrifugation at $12,000 \mathrm{x}$ g for $12 \mathrm{~min}$ at $4^{\circ} \mathrm{C}$ and the supernatant $(500 \mathrm{ml}$ per reaction) were incubated with biotin-labeled wild-type or mutant miR-3182, followed by incubation with M-280 streptavidin beads (100 $\mu$; Sigma-Aldrich; Merck KGaA) at $4^{\circ} \mathrm{C}$ overnight. The beads were washed twice with cold lysis buffer and precipitated RNAs were eluted using elution buffer (Sigma-Aldrich; Merck KGaA) according to the manufacturer's protocol, followed by RT-qPCR analysis as described above. Transfection conditions were the same as described above.

Luciferase reporter assay. The bioinformatics online tools miRDB (http://mirdb.org/miRDB/index.html) and TargetScan 7 (http://www.targetscan.org/vert_71/) predicted that miR-3182 had targeted binding sites with lncRNA DUXAP8 and NUPR1. The 3'UTR of DUXAP8 and NUPR1 containing binding site sequence was amplified and constructed into psiCHECK2 luciferase reporter vector (Promega Corporation), referred to as DUXAP8-WT and NUPR1-WT reporter plasmids, respectively. The mutant (MUT) sequence was constructed on the reporter gene vector and recorded as DUXAP8-MUT and NUPR1-MUT reporter plasmids. A375 and A2058 cells were transfected with indicated luciferase reporter and miR-3182 mimic (5'-GCUUCUGUAGUGUAGUC3'-) or NC mimic (5'-UUCUCCGAACGUGUCACGUTT-3') (Shanghai GenePharma Co., Ltd.) using Lipofectamine ${ }^{\circledR} 2000$ reagent (Invitrogen, Thermo Fisher Scientific, Inc.) for $48 \mathrm{~h}$ and then luciferase reporter assay was performed using a Dual Luciferase Reporter Assay kit (Promega Corporation) according to according to the manufacturer's protocol. Dual luciferase activity was measured separately, and the relative luciferase activity of each cell was calculated based on the fluorescence intensity and normalized to Renilla luciferase activity.

Statistical analysis. Statistical analysis was carried out using SPSS 22.0 software (IBM Corp.). Continuous data ware expressed as the mean \pm standard deviation. Differences between groups were compared using an unpaired Student's t-test or one-way ANOVA followed by Tukey's post hoc test. The prognosis of patients was analyzed using the Kaplan-Meier method and log-rank tests. The relationship between DUXAP8 expression and miR-3182 or NUPR1 levels was analyzed using Pearson's correlation coefficient. $\mathrm{P}<0.05$ was considered to indicate a statistically significant difference.

\section{Results}

Expression of DUXAP8 and survival analysis. The expression levels of lncRNA DUXAP8 were examined in melanoma tissue samples and cell lines. DUXAP8 was upregulated in melanoma tissue compared with adjacent normal tissue (Fig. 1A). Moreover, patients with stage III+IV melanoma displayed higher levels of DUXAP8 than patients in stage I-II (Fig. 1B). Similar results were also observed in cell lines. The expression of DUXAP8 in malignant melanoma cell lines (A375, A2058 and SK-MEL-2) was higher than that in HEMa-LP cells (Fig. 1C). A375 and A2058 cell lines displayed the highest levels of DUXAP8. Therefore, these two cell lines were used in subsequent experiments. In addition, the patients were divided into two groups based on the median value of 
A

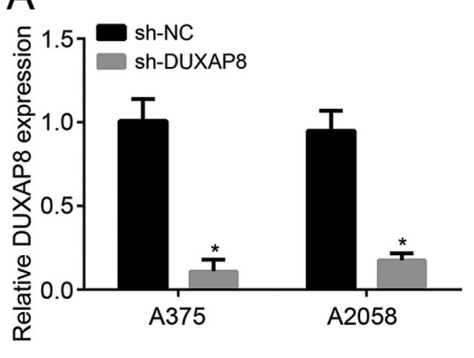

B

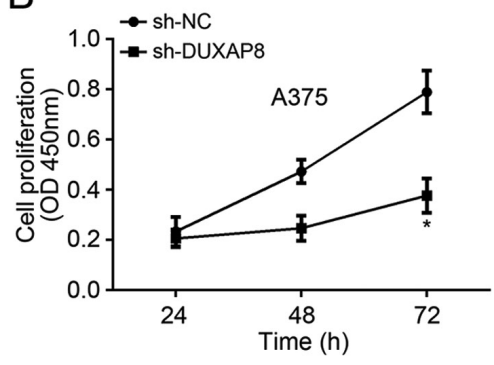

C
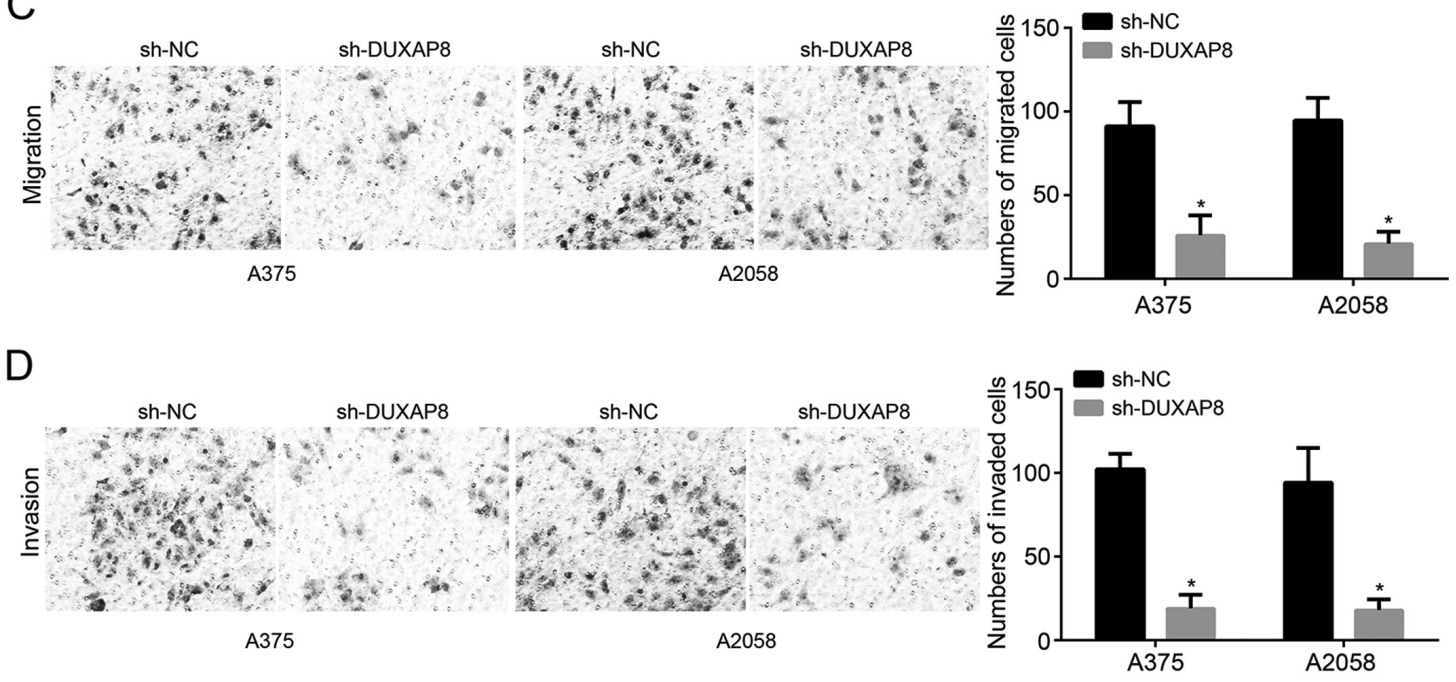

Figure 2. Knockdown of DUXAP8 inhibits progression of melanoma cells. (A) shRNA-mediated knockdown of DUXAP8. (B) CCK-8 analysis of proliferation following sh-DUXAP8 transfection. (C and D) Transwell assay for (C) migration and (D) invasion analysis following sh-DUXAP8 transfection (magnification, x100). "P<0.05. DUXAP8, double homeobox A pseudogene 8; shRNA, short hairpin RNA; OD, optical density; NC, negative control.

DUXAP8 expression, and patients with higher expression of DUXAP8 experienced shorter survival time (Fig. 1D). High DUXAP8 levels were associated with poor prognosis.

DUXAP8 knockdown inhibits the migration and invasion of melanoma cells. The expression of DUXAP8 was significantly reduced following transfection with sh-DUXAP8, which confirmed the transfection was successful (Fig. 2A). In order to examine the effect of sh-DUXAP8 on proliferation, CCK-8 assays were carried out. After transfection for $48 \mathrm{~h}$ and further culturing for $72 \mathrm{~h}$, the proliferation of A375 and A2058 cells significantly decreased in the sh-DUXAP8 group compared with that of the sh-NC group (Fig. 2B). In addition, DUXAP8 knockdown significantly decreased migration and invasion of melanoma cells (Fig. 2C and D). Therefore, DUXAP8 knockdown inhibited the proliferation, migration and invasion of melanoma cells.

IncRNA DUXAP8 targets miR-3182, whereas miR-3182 targetsNUPR1. To identify the potential targets of DUXAP8, bioinformatics analysis was carried out using miRDB. miR-3182 was identified as the most likely target according to the prediction results (Fig. 3A). To validate the interaction between DUXAP8 and miR-3182, a dual luciferase reporter assay was carried out. Both in A375 and A2058 cell lines, transfection of miR-3182 reduced the luciferase activity in the DUXAP8-WT group. However, NC miRNA did not significantly reduce the luciferase activity of the vector. In the DUXAP8-MUT group, luciferase activity remained unchanged. These results indicated that miR-3182 significantly downregulated luciferase activity (Fig. 3B and C). Pull-down assays also demonstrated that miR-3182-WT precipitated DUXAP8, proving their binding (Fig. 3D). Following DUXAP8 knockdown, the expression of miR-3182 significantly increased (Fig. 3E). To identify the targets of miR-3182, bioinformatics analysis was performed using TargetScan7. The results indicated that NUPR1 was the most likely candidate. The predicted binding sites of miR-3182 and NUPR1 are shown in Fig. 3F. Moreover, luciferase reporter assays confirmed this interaction (Fig. 3G and H). Transfection with miR-3182 downregulated the expression of NUPR1 in both A375 and A2058 cells (Fig. 3I). More importantly, sh-DUXAP8 decreased the expression of NUPR1, while the miR-3182 inhibitor reversed this effect (Fig. 3J and K). Thus, DUXAP8 promotes NUPR1 expression by inhibiting miR-3182.

Rescue assay and correlation analysis. For rescue assays, NUPR1 was overexpressed in A375 cells and A2058 cells (Fig. 1A). CCK8 and transwell assays demonstrated that the cell proliferation, migration and invasion were inhibited in the sh-DUXAP8+miR-NC+pcDNA3-vector group compared with the sh-NC+miR-NC+pcDNA3-vector group (Fig. 4A-C). However, proliferation, migration and invasion were rescued by NUPR1 overexpression in sh-DUXAP8+miR-NC+oeNUPR1 group compared with that in the sh-DUXAP8+miR-NC+pcDNA3-vector group (Fig. 4A-C). 
A

\begin{tabular}{l|c|c|c|}
\multicolumn{2}{l}{ miRDB result: } \\
$\begin{array}{c}\text { Target } \\
\text { Detail }\end{array}$ & $\begin{array}{c}\text { Target } \\
\text { Score }\end{array}$ & miRNA Name & Gene Symbol \\
\hline \hline Details & 86 & hsa-miR-3182 & submission \\
\hline
\end{tabular}
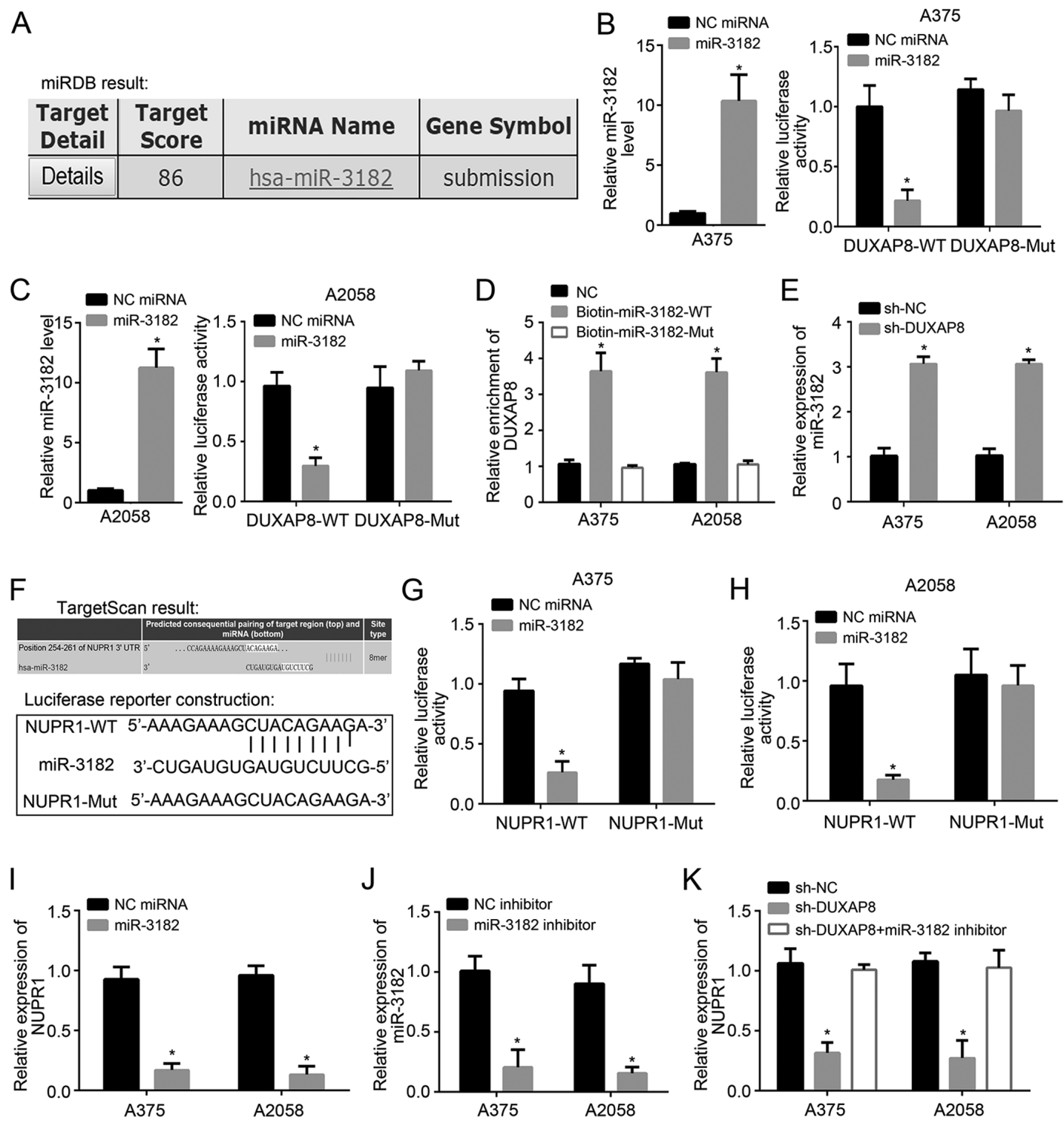

Figure 3. DUXAP8 targets miR-3182, while miR-3182 targets NUPR1. (A) DUXAP8 target prediction results from the miRDB online tool. (B and C) Luciferase reporter assays confirming that DUXAP8 binds to miR-3182. (D) RNA pull-down analysis of the binding of DUXAP8 to miR-3182. (E) miR-3182 expression levels following sh-DUXAP8 transfection. (F) TargetScan prediction of the binding site between miR-3182 and NUPR1. (G and H) Luciferase assays confirming that miR-3182 binds to NUPR1. (I) NUPR1 mRNA expression following transfection. (J) Analysis of miR-3182 expression following transfection. (K) Analysis of NUPR1 expression following transfection. "P<0.05. DUXAP8, double homeobox A pseudogene 8; NUPR1, nuclear protein 1 transcriptional regulator; miR/miRNA, microRNA; shRNA, short hairpin RNA; NC, negative control; hsa, Homo sapiens; WT, wild-type; Mut, mutant.

Proliferation,migrationandinvasionweresuppressedbymiR-3182 in the sh-NC+miR-3182+pcDNA3-vector group compared with the sh-NC+miR-NC+pcDNA3-vector group (Fig. 4A-C). Proliferation, migration and invasion were also rescued by NUPR1 overexpression in sh-NC+miR-3182+oeNUPR1 group compared with the sh-NC+miR-3182+pcDNA3-vector group (Fig. 4A-C). In melanoma tissue samples, DUXAP8 expression negatively correlated with that of miR-3182, but positively correlated with that of NUPR1 (Fig. 4D and E). Altogether, these results confirmed the regulatory relationship between DUXAP8, miR-3182 and NUPR1.

\section{Discussion}

In order to examine the role of DUXAP8 in melanoma, DUXAP8-related miRNA and genes were explored. In the present study, DUXAP8 was upregulated in melanoma tissue samples and cell lines. DUXAP8 levels were negatively associated with the survival time of patients with melanoma. DUXAP8 knockdown inhibited the proliferation, migration and invasion of melanoma cells.

Previous studies have shown that lncRNA derived from pseudogenes could also regulate gene expression through various mechanisms, such as binding to RNA-binding proteins and regulating target genes $(18,19)$. Xu et al $(20)$ carried out a genome-wide analysis and identified the lncRNA DUXAP8 as a significant biomarker of metastatic renal carcinoma. Ma et al (19) confirmed that DUXAP8 downregulated pleckstrin homology domain containing $\mathrm{O} 1$ level and promoted the progression of gastric cancer. Moreover, DUXAP8 could also inhibit Ras homolog family member B (RHOB) and early growth response 1 (EGR1) expression and accelerate 

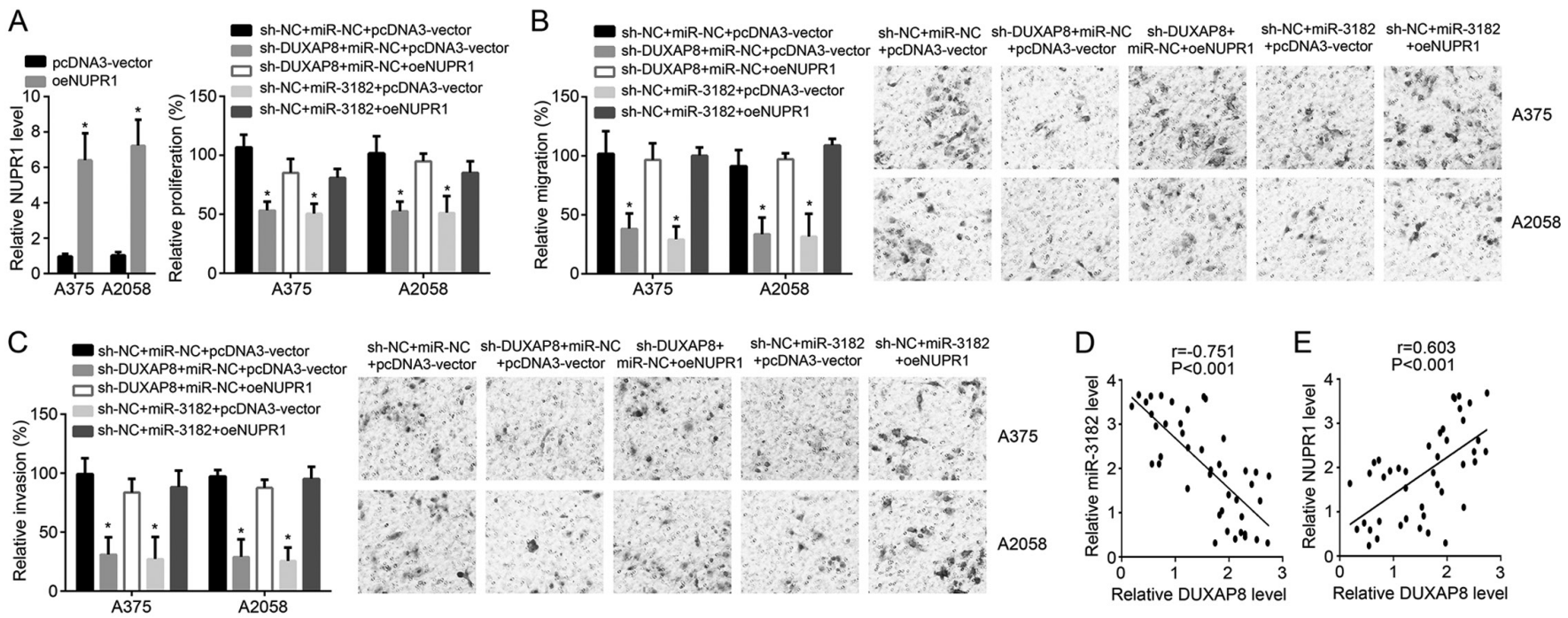

Figure 4. Rescue assay and correlation analysis. (A) CCK8 assay for proliferation. The absorbance was measured after $72 \mathrm{~h}$. (B and C) Transwell experiments for detecting (B) migration and (C) invasion (magnification, x100). (D) Correlation analysis of miR-3182 and DUXAP8 in tumor tissues. (E) Correlation analysis of NUPR1 and DUXAP8. "P<0.05. DUXAP8, double homeobox A pseudogene 8; NUPR1, nuclear protein 1 transcriptional regulator; miR/miRNA, microRNA; shRNA, short hairpin RNA; NC, negative control; oe, overexpression.

development of lung cancer in vitro (18). Although the role of DUXAP8 in melanoma has not been reported so far, the effects of DUXAP8-related genes have been confirmed. Indeed, as reported in a previous study, the RHO family is important for the activation of the FAK signaling pathway, which increases aggregation of melanoma cells (21). Importantly, the expression of RHOB is closely related to drug resistance in melanoma cells (22). Furthermore, RHOB also regulates the RAS/PI3K/AKT pathway, which increases invasion and migration of melanoma cells and patient survival (23). Besides, the transcription factor EGR1 was differentially expressed in saliva samples of mice with melanoma compared with normal tissues (24). Based on the aforementioned evidence, it may be hypothesized that DUXAP8 plays an important role in the development of melanoma.

Bioinformatics prediction and in vitro experiments confirmed that DUXAP8 targeted miR-3182 in this study. miR-3182 is involved in different diseases, such as breast cancer (25), osteosarcoma (26) and lung cancer (27). Zhou et al (28) proposed that miR-3182 was important for metastasis of nasopharyngeal carcinoma and prognosis of this disease. In breast cancer, miR-3182 can inhibit mTOR signaling (25). It is well known that the mTOR signaling pathway is closely associated with autophagy, invasion and proliferation of melanoma cell (29). The effect of miR-3182 in melanoma cells could not be ignored. In the present study, DUXAP8 promoted melanoma development by downregulating the expression of miR-3182.

Furthermore, miR-3182 was found to target NUPR1 in this study. In previous studies, NUPR1 has been confirmed to be associated with lung cancer, pancreatic cancer and liver cancer (30-32). In non-small cell lung cancer, knockdown of NUPR1 inhibited proliferation in vitro and slowed tumor growth in vivo (30). Emma et al (32) reported that NUPR1 regulated RUNX2 expression and further effect activation of hepatocellular carcinoma cells. Interestingly, RUNX2 was upregulated in melanoma tumor cells compared with controls and was also involved in migration of cells (33). In malignant tumors, NUPR1 was confirmed to modulate the caspase 3 and PTEN levels and also regarded as a novel target for treatment (32). In addition, knockdown of PTEN is critical for the progression of melanoma development (34). In the present study, the direct effect of NUPR1 on melanoma cell lines was examined. Overexpression of NUPR1 reversed the effect of DUXAP8 knockdown or miR-3182 mimic transfection on melanoma cells. In melanoma tissue, DUXAP8 expression positively correlated with that of NUPR1. Therefore, NUPR1 is critical factor in the function of DUXAP8 in proliferation, invasion and migration of melanoma.

In conclusion, the IncRNA DUXAP8 may accelerate the development of melanoma by regulating miR-3182/NUPR1 expression. This demonstrates the molecular function of the DUXAP8/miR-3182/NUPR1 axis in melanoma progression and suggests these molecules may represent potential targets for melanoma treatment.

\section{Acknowledgements}

Not applicable.

\section{Funding}

No funding was received.

\section{Availability of data and materials}

The datasets used and/or analyzed during the current study are available from the corresponding author on reasonable request.

\section{Authors' contributions}

XC and NL designed the study. XC, JG and NL contributed toward performing the experiments, data analysis, drafting 
and critically revising the paper, gave final approval of the version to be published, and confirm the authenticity of all the raw data. All authors read and approved the final manuscript.

\section{Ethics approval and consent to participate}

This study was approved by The Ethics Committee of The Affiliated Changzhou No. 2 People's Hospital with Nanjing Medical University (Changzhou, China). All patients signed informed consent forms.

\section{Patient consent for publication}

Not applicable.

\section{Competing interests}

The authors declare that they have no competing interests.

\section{References}

1. Fodstad $\mathrm{O}$, Aass $\mathrm{N}$ and Pihl A: An inverse relationship between the growth rate of human melanoma xenografts and their response to some cytostatic drugs. Br J Cancer 41: 829-831, 1980.

2. Farahmand AM, Ehsani AH, Mirzaei M, Mohsenian M and Ghanadan A: Patients' characteristics, histopathological findings, and tumor stage in different types of malignant melanoma: A retrospective multicenter study. Acta Med Iran 55: 316-323, 2017.

3. Hu DN, Yu GP and McCormick SA: Population-based incidence of vulvar and vaginal melanoma in various races and ethnic groups with comparisons to other site-specific melanomas. Melanoma Res 20: 153-158, 2010.

4. Del Vecchio M: AACR update on 5-year survival rates, effcacy and long-term safety in previously treated advanced/metastatic melanoma patients receiving mono-immunotherapy with nivolumab. Recenti Prog Med 107: 414-417, 2016 (In Italian).

5. Morton DL, Davtyan DG, Wanek LA, Foshag LJ and Cochran AJ: Multivariate analysis of the relationship between survival and the microstage of primary melanoma by Clark level and Breslow thickness. Cancer 71: 3737-3743, 1993.

6. Pfahlberg A, Kölmel KF and Gefeller O; Febim Study Group: Timing of excessive ultraviolet radiation and melanoma: Epidemiology does not support the existence of a critical period of high susceptibility to solar ultraviolet radiation-induced melanoma. Br J Dermatol 144: 471-475, 2001.

7. Thoelke A, Willrodt S, Hauschild A and Schadendorf D: Primary extracutaneous malignant melanoma: A comprehensive review with emphasis on treatment. Onkologie 27: 492-499, 2004.

8. Gasparini G: Tamoxifen and chemotherapy in the treatment of metastatic melanoma: Are there other possible mechanisms explaining their potentiation? J Clin Oncol 12: 1994-1996, 1994.

9. Wang X, Moschos SJ and Becker D: Functional analysis and molecular targeting of aurora kinases a and B in advanced melanoma. Genes Cancer 1: 952-963, 2010.

10. Chi Z, Li S, Sheng X, Si L and Guo J: Clinical presentation, histology, and prognoses of malignant melanoma in ethnic Chinese: A study of 522 consecutive cases. BMC Cancer 11: 85, 2011.

11. Barth A, Wanek L and Morton DL: Prognostic factors in 1,521 melanoma patients with distant metastases. J Am Coll Surg 181: 193-201, 1995

12. Ribero S, Glass D and Bataille V: Genetic epidemiology of melanoma. Eur J Dermatol 26: 335-339, 2016.

13. Schmidt K, Joyce CE, Buquicchio F, Brown A, Ritz J, Distel RJ, Yoon $\mathrm{CH}$ and Novina CD: The lncRNA SLNCR1 mediates melanoma invasion through a conserved SRA1-like region. Cell Rep 15: 2025-2037, 2016.

14. Gong J, Zhang H, He L, Wang L and Wang J: Increased expression of long non-coding RNA BCAR4 is predictive of poor prognosis in patients with non-small cell lung cancer. Tohoku J Exp Med 241: 29-34, 2017.
15. Wei F, Yang L, Jiang D, Pan M, Tang G, Huang M and Zhang J: Long noncoding RNA DUXAP8 contributes to the progression of hepatocellular carcinoma via regulating miR-422a/PDK2 axis. Cancer Med 9: 2480-2490, 2020.

16. Ji X, Tao R, Sun LY, Xu XL and Ling W: Down-regulation of long non-coding RNA DUXAP8 suppresses proliferation, metastasis and EMT by modulating miR-498 through TRIM44-mediated $\mathrm{AKT} / \mathrm{mTOR}$ pathway in non-small-cell lung cancer. Eur Rev Med Pharmacol Sci 24: 3152-3165, 2020.

17. Livak KJ and Schmittgen TD: Analysis of relative gene expression data using real-time quantitative PCR and the 2(-Delta Delta C(T)) method. Methods 25: 402-408, 2001.

18. Sun M, Nie FQ, Zang C, Wang Y, Hou J, Wei C, Li W, He X and $\mathrm{Lu} \mathrm{KH}$ : The pseudogene DUXAP8 promotes non-small-cell lung cancer cell proliferation and invasion by epigenetically silencing EGR1 and RHOB. Mol Ther 25: 739-751, 2017.

19. Ma HW, Xie M, Sun M, Chen TY, Jin RR, Ma TS, Chen QN, Zhang EB, He XZ, De W and Zhang ZH: The pseudogene derived long noncoding RNA DUXAP8 promotes gastric cancer cell proliferation and migration via epigenetically silencing PLEKHO1 expression. Oncotarget 8: 52211-52224, 2016.

20. Xu X, Xu Y, Shi C, Wang B, Yu X, Zou Y and Hu T: A genome-wide comprehensively analyses of long noncoding RNA profiling and metastasis associated lncRNAs in renal cell carcinoma. Oncotarget 8: 87773-87781, 2017.

21. Goundiam O, Nagel MD and Vayssade M: Akt and RhoA inhibition promotes anoikis of aggregated B16F10 melanoma cells. Cell Biol Int 36: 311-319, 2012.

22. Delmas A, Cherier J, Medale-Giamarchi C, Pradines A and Favre G: Abstract 3401: Vemurafenib-induced RhoB expression leads to melanoma cell resistance. Cancer Res 73 (Suppl 8): S3401, 2013.

23. Eide A: The role of the sub-commission on promotion and protection of human rights and its working groups in the prevention of conflicts. Int J Minor Group Rights 8: 25-29, 2001

24. Gao K, Zhou H, Zhang L, Lee JW, Zhou Q, Hu S, Farrell J, Eibl G and Wong D: Abstract \#314: Induction of systemic disease-specific salivary biomarker profiles in mouse models of melanoma and non-small cell lung cancer. Cancer Res 69 (Suppl 9): S314, 2009.

25. Razaviyan J, Hadavi R, Tavakoli R, Kamani F, Paknejad M and Mohammadi-Yeganeh S: Expression of miRNAs targeting mTOR and S6K1 genes of mTOR signaling pathway including miR-96, miR-557, and miR-3182 in triple-negative breast cancer. Appl Biochem Biotechnol 186: 1074-1089, 2018.

26. Zhu KP, Ma XL and Zhang CL: LncRNA ODRUL contributes to osteosarcoma progression through the miR-3182/MMP2 axis. Mol Ther 25: 2383-2393, 2017.

27. Xue M, Shi D, Xu G and Wang W: The long noncoding RNA linc00858 promotes progress of lung cancer through miR-3182/MMP2 axis. Artif Cells Nanomed Biotechnol 47: 2091-2097, 2019.

28. Zhou MY, Lan GP, Si YF and Mo XG: Expression patterns and significances of distant metastasis and prognosis of MiR-3182 innasopharygeal carcinoma. Chin J New Clin Med 5: 369-372, 2016.

29. Zhao G, Han X, Zheng S, Li Z, Sha Y, Ni J, Sun Z, Qiao S and Song Z: Curcumin induces autophagy, inhibits proliferation and invasion by downregulating AKT/mTOR signaling pathway in human melanoma cells. Oncol Rep 35: 1065-1074, 2016.

30. Guo X, Wang W, Hu J, Feng K, Pan Y, Zhang L and Feng Y: Lentivirus-mediated RNAi knockdown of NUPR 1 inhibits human nonsmall cell lung cancer growth in vitro and in vivo. Anat Rec (Hoboken) 295: 2114-2121, 2012.

31. Cano CE, Hamidi T, Garcia MN, Grasso D, Loncle C, Garcia S, Calvo E, Lomberk G, Dusetti N, Bartholin L, et al: Genetic inactivation of Nuprl acts as a dominant suppressor event in a two-hit model of pancreatic carcinogenesis. Gut 63: 984-995, 2014.

32. Emma MR, Iovanna JL, Bachvarov D, Puleio R, Loria GR, Augello G, Candido S, Libra M, Gulino A, Cancila V, et al: NUPR1, a new target in liver cancer: Implication in controlling cell growth, migration, invasion and sorafenib resistance. Cell Death Dis 7: e2269, 2016.

33. Boregowda RK, Olabisi OO, Abushahba W, Jeong BS, Haenssen KK, Chen W, Chekmareva M, Lasfar A, Foran DJ, Goydos JS and Cohen-Solal KA: RUNX2 is overexpressed in melanoma cells and mediates their migration and invasion. Cancer Lett 348: 61-70, 2014.

34. $\mathrm{Wu} \mathrm{H}$, Goel $\mathrm{V}$ and Haluska FG: PTEN signaling pathways in melanoma. Oncogene 22: 3113-3122, 2003. 